Artículos

\title{
Características del aprendizaje autónomo en estudiantes de kinesiología de una institución de educación superior de Buenos Aires*
}

\author{
Characteristics of the Autonomous Learning in Kinesiology Students in a Buenos Aires College Caraterísticas da \\ aprendizagem autónoma em alunos de cinesiologia de uma instituição de ensino superior em Buenos Aires
}

\author{
Carlos Canova Barrios ${ }^{\text {a }}$ \\ Universidad de Ciencias Empresariales y Sociales, \\ Argentina \\ carlos.canova1993@gmail.com \\ ORCID: http://orcid.org/0000-0003-3901-6117
}

Luis Pecker

Universidad de Ciencias Empresariales y Sociales,

Argentina

ORCID: http://orcid.org/0000-0003-3209-7727
DOI: https://doi.org/10.11144/Javeriana.ie21-2.caae

Fecha de recepción: 27 Julio 2018

Fecha de aprobación: 20 Junio 2019

Fecha de publicación: 30 Noviembre 2019

\section{Resumen:}

Introducción: las estrategias de trabajo autónomo cobran cada vez más relevancia en los niveles medio y superior de la educación, sobre todo con los cambios que actualmente se viven en el sistema educativo, que hacen hincapié en la autonomía y en la responsabilidad del alumno en la gestión de su aprendizaje. Objetivo: identificar las características del aprendizaje autónomo en los estudiantes de kinesiología de una institución de educación superior de la ciudad de Buenos Aires, Argentina. Metodología: estudio descriptivo, de corte transversal y con enfoque cuantitativo. Se implementó el Cuestionario de Estrategias de Trabajo Autónomo. Resultados: muestra conformada por 95 estudiantes de la carrera de Licenciatura en Kinesiología y Fisiatría, de esta el 43\% era de sexo femenino, con edad promedio de 25,6 años (DE: 5,6). Se encontró un bajo uso de las estrategias de planificación y un amplio desarrollo de las de preparación de exámenes. Conclusión: los estilos de aprendizaje pueden ser de gran ayuda para mejorar el rendimiento académico, y es necesario dentro de la gestión educativa considerar diagnosticar y conocer el estilo predilecto para adquirir y procesar la información por parte del estudiantado. Palabras clave: estudiantes, educación superior, aprendizaje, autonomía.

\section{Abstract:}

Introduction: Autonomous work strategies are increasingly relevant in the intermediate and higher education, even more with the changes currently being developed in the educative system that emphasize the student's autonomy and responsibility regarding his/ her learning process. Objective: To identify the characteristics of the autonomous learning in kinesiology students from Buenos Aires college, Argentina. Methodology: It is a cross-sectional descriptive study with a quantitative approach. The study used the Autonomous Work Strategy Questionnaire. Results: From the sample consisting of 95 students in the Kinesiology and Physiatry Program, $43 \%$ were women and the mean age was 25.6 years (SD: 5.6). The study found a low use of planning strategies and a broad development of strategies for test preparation. Conclusion: Learning styles can be greatly useful to enhance the academic performance. In the educational management studied herein, there is a need to consider, diagnose, and know those styles the students prefer to get and process the information.

Keywords: students, higher education, learning, autonomy.

\section{Resumo:}

Introdução: As estratégias de trabalho autônomo estão se tornando cada vez mais relevantes nos níveis médio e superior do ensino, sobre todo com as mudanças atualmente ocorridas no sistema educativo, que enfatizam a autonomia e responsabilidade do aluno na gestão da sua aprendizagem. Objetivo: Identificar as caraterísticas da aprendizagem autônoma nos alunos de cinesiologia de uma instituição de ensino superior da cidade de Buenos Aires, Argentina. Metodologia: Estudo descritivo transversal e com abordagem

Notas de autor

a Autor de correspondencia. Correo electrónico: carlos.canova1993@gmail.com 
quantitativa. O Questionário de Estratégias de Trabalho Autônomo foi implementado. Resultados: Amostra conformada por 95 alunos da carreira de Licenciatura em cinesiologia e Fisiatria, da qual 43\% eram de sexo feminino, com idade média de 25,6 anos (DE: 5,6). Encontrou-se baixo uso de estratégias de planejamento e um amplo desenvolvimento das de preparação de exames. Conclusão: os estilos de aprendizagem podem ser de grande ajuda para melhorar o desempenho académico, e é preciso dentro da gestão educativa, considerar diagnosticar e conhecer o estilo predileto para adquirir e processar a informação por parte do alunato.

Palavras-chave: alunos, ensino superior, aprendizagem, autonomia.

\section{Introducción}

Clemente Lobato definió el aprendizaje autónomo como la

modalidad de aprendizaje en la que el estudiante se responsabiliza de la organización de su trabajo y de la adquisición de las diferentes competencias según su propio ritmo, etc. Implica asumir la responsabilidad y el control del proceso personal de aprendizaje, y las decisiones sobre la planificación, realización y evaluación de la experiencia de aprendizaje. (1)

Las estrategias de trabajo autónomo cobran cada vez más relevancia en los niveles medio y superior de la educación, sobre todo con los cambios que actualmente se viven en el sistema educativo, que hacen hincapié en la autonomía y la responsabilidad del alumno en la gestión de su aprendizaje y la construcción del propio conocimiento, de modo que le permita afrontar con éxito las necesidades emergentes de los sectores de trabajo, necesarias para el correcto desempeño de su futuro rol profesional $(2,3)$.

A partir de lo anterior, John Dewey considera el aprendizaje algo que hace la persona cuando estudia. Esto implica la participación del estudiante en más que la escucha pasiva y un desplazamiento gradual del sistema de aprendizaje pasivo a un desarrollo de habilidades en el alumnado, desde lo cual se realicen tareas que requieren cierto grado de participación, pensamiento complejo e, incluso, el cuestionamiento de las propias creencias y valores para conseguir cambios o mejoras de conducta (4). Por lo anterior, el aprendizaje es una acción que toma la información disponible y genera nuevo conocimiento (5).

Sobre la base de estas ideas puede entenderse la docencia como un modo de formar profesionales competentes, capaces de gestionar y responsabilizarse de su propio conocimiento, maximizando su perfil de análisis crítico e intelectual en general, fomentando su desarrollo personal y profesional, no solo durante el proceso de formación académico-universitario, sino durante toda su vida.

Todo lo anterior es relevante en el quehacer del profesional sanitario, porque prima la certeza de que cada paciente es único y debe ser manejado de manera individual. De ahí que el aprendizaje autónomo sea un medio para fomentar habilidades de pensamiento que se traducen en mejor calidad del cuidado prestado al paciente en todas las etapas por las que atraviese, y entendiéndose que sería difícil imaginar a un alumno adquiriendo competencias complejas de aprendizaje y llevando a cabo procesos de calidad asistencial, gerencial, investigativas y de intervención comunitaria, si no dispone de un repertorio de estrategias activas necesarias para conseguirlo. En otras palabras, el aprendizaje memorístico es un proceso cognitivo básico, y en el caso de las ciencias de la salud, los estudiantes deben desarrollar estrategias de aprendizaje autorregulado y complejo (6).

El propósito del presente trabajo fue identificar las características del aprendizaje autónomo en los estudiantes de kinesiología de una institución de educación superior de la ciudad de Buenos Aires, Argentina.

\section{Materiales y métodos}

Tipo de estudio: estudio descriptivo de corte transversal, con enfoque cuantitativo.

Población y muestra: la población estuvo conformada por los estudiantes de la carrera de Licenciatura en Kinesiología y Fisiatría de una institución de educación superior de la ciudad de Buenos Aires, Argentina. 
El muestreo fue intencional y la muestra la constituyeron 95 estudiantes que accedieron voluntariamente a hacer parte del presente estudio. A ellos se les solicitó su participación en las aulas de clases, mediante la firma del consentimiento informado.

Criterios de inclusión y exclusión: como criterios de inclusión se enmarcaron ser estudiante activo de la carrera de licenciatura en Kinesiología y Fisiatría, y acceder voluntariamente a ser parte del estudio. Como criterios de exclusión se consideraron no acceder a ser parte de la investigación y contar con algún impedimento para el diligenciamiento del cuestionario (por ejemplo, discapacidades: no vidente).

Recolección de la información: se aplicó una encuesta autoadministrada a los estudiantes en las aulas. Esta encuesta estaba constituida por dos partes: la primera, que evaluó el perfil sociodemográfico del participante (sexo, edad, cuatrimestre que cursa actualmente, estado civil, tener hijos y cuántos) y promedio general de la carrera en el momento del estudio, y la segunda parte, que midió las características del aprendizaje autónomo. Para esta segunda parte, se implementó el Cuestionario de Estrategias de Trabajo Autónomo (CETA), la cual cuenta con alta consistencia interna e índices alfa de Cronbach de 0,898 . Este constructo está dividido en seis subescalas que han alcanzado valores aceptables de fiabilidad alfa de Cronbach (7). Está constituida por 45 ítems que se valoran en una escala tipo Likert, donde el sujeto evalúa en una escala de 5 adjetivos que van desde nunca (1) hasta siempre (5) en forma de afirmaciones sobre las estrategias de trabajo autónomo habitualmente utilizadas.

Corrección y análisis de la prueba: para la corrección de la prueba se sumaron los ítems correspondientes a las estrategias, divididas entre el número de ítems totales sumados. El puntaje arrojado muestra el promedio de uso de dichas estrategias en los estudiantes. A continuación, se relacionan las estrategias y sus ítems de evaluación:

Factor Estrategias de Ampliación. Este describe las estrategias relacionadas con la búsqueda y elaboración de materiales y actividades complementarias de ampliación del material propuesto por el profesor. Se evalúa por medio de la sumatoria de las preguntas $8,9,14,16,18,19,25,40$ y 45, y luego dividiéndolo entre las 9 preguntas que conforman el factor.

Factor Estrategias de Colaboración. Este describe una serie de estrategias relacionadas con la implicación del alumno en tareas grupales, y sobre cómo se relaciona con otros compañeros en tareas relacionadas con el aprendizaje. Se evalúa por medio de la sumatoria de las preguntas 15, 21, 22, 23, 24, 26, 27, 31, 33, 37 y 38 , y luego dividiéndolo entre las 11 preguntas que conforman el factor.

Factor Estrategias de Conceptualización. Este reúne estrategias relacionadas con el trabajo intelectual sobre el contenido; incluyen tareas como elaboración de esquemas, resúmenes, mapas conceptuales, etc. Se evalúa por medio de la sumatoria de las preguntas $3,5,6,7,11,13,32$ y 43 , y luego dividiéndolo entre las 8 preguntas que conforman el factor.

Factor Estrategias de Planificación. Este recoge aspectos relativos a la planificación de tiempos y programación de las tareas, tanto de estudio como de elaboración de trabajos, así como un ítem relacionado con la evaluación de los procedimientos de aprendizaje. Se evalúa por medio de la sumatoria de las preguntas 10, 12, 17, 39 y 44, y luego dividiéndolo entre las 5 preguntas que conforman el factor.

Factor Estrategias de Preparación de Exámenes. Este se encuentra relacionado con las estrategias implementadas para la preparación ante exámenes. Se evalúa por medio de la sumatoria de las preguntas 20, 29, 34, 35, 36 y 42, y luego dividiéndolo entre las 6 preguntas que conforman el factor.

Factor Estrategias de Participación. Describe el nivel de participación del alumno, analizando aspectos como la asistencia a clase, aclaración de dudas, participación en el aula o tutorías. Se evalúa por medio de la sumatoria de las preguntas 1, 2, 4, 28, 30 y 41, y luego dividiéndolo entre las 6 preguntas que conforman el factor.

Estadística: se usó para el análisis de la información obtenida la versión 23 del programa estadístico SPSS. Los resultados son mostrados en tablas de distribución de frecuencia. Para determinar si existen diferencias 
en las estrategias en función del sexo, año de cursada, tenencia de hijos, cantidad de horas de trabajo y notas, se realizó un análisis simple de la varianza (Anova).

Ética: previamente, se obtuvo el consentimiento informado de los participantes. La confidencialidad de los datos fue protegida en cumplimiento de la Ley 25326 de Protección de los Datos Personales de la Dirección Nacional de Protección de los Datos Personales del Ministerio de Justicia, Seguridad y Derechos Humanos de Argentina. Se respetaron las disposiciones internacionales sobre Buena Práctica en Investigación en Seres Humanos, adhiriéndonos a la Declaración de Helsinki. Se les aseguró que los datos recolectados se analizarían de manera grupal y que la información se solicitaba solo con fines académicos. El diligenciamiento del constructo no requería número de documento, matrícula, nombre o algún otro dato que pudiese usarse para conectar al estudiante con la encuesta respondida; así, se aseguró la privacidad y la seguridad en el uso de los datos suministrados. La participación fue en todo momento voluntaria y el estudiante podía retirarse en cualquier momento. Los autores de la investigación tienen publicado el cuestionario CETA para su uso y se solicitó el permiso correspondiente, del cual se obtuvo su aprobación para el uso en el presente trabajo.

\section{Resultados}

\section{Caracterización de la muestra}

La muestra estuvo conformada por 95 estudiantes de la Licenciatura en Kinesiología y Fisiatría, de esta el $43 \%$ era de sexo femenino, con edades comprendidas entre los 18 y los 50 años, y cuya edad promedio fue de 25,6 años (DE: 5,6). El 70\% eran solteros y el 66,3\% se encontraba trabajando, con un promedio de 35 horas de trabajo semanales (DE: 9). El 7,3\% tiene hijos.

\section{Estrategias de trabajo autónomo}

En la tabla 1 se muestra el análisis de la distribución de las estrategias de trabajo autónomo. La estrategia más usada fue la de preparación de exámenes con una media de 3,6 (DE: 0,7) y la menos implementada es la estrategia de planificación con una media de 2,6 (DE: 0,8).

TABLA 1.

Estrategias de trabajo autónomo

\begin{tabular}{lrrrrrr}
\hline Medida & $\begin{array}{r}\text { Estrategias } \\
\text { de } \\
\text { ampliación }\end{array}$ & $\begin{array}{r}\text { Estrategias de } \\
\text { colaboración }\end{array}$ & $\begin{array}{r}\text { Estrategias de } \\
\text { conceptualización }\end{array}$ & $\begin{array}{r}\text { Estrategias de } \\
\text { planificación }\end{array}$ & $\begin{array}{r}\text { Estrategias de } \\
\text { preparación } \\
\text { de exámenes }\end{array}$ & $\begin{array}{r}\text { Estrategias de } \\
\text { participación }\end{array}$ \\
\hline Media & 3,2 & 2,8 & 3,0 & 2,6 & 3,6 & 3,2 \\
\hline Mediana & 3,3 & 2,9 & 3,1 & 2,6 & 3,7 & 3,3 \\
\hline Moda & 3,8 & 2,7 & 3,6 & 2,4 & 3,7 & 3,3 \\
\hline $\begin{array}{l}\text { Desviación } \\
\text { estándar }\end{array}$ & 0,6 & 0,7 & 0,7 & 0,8 & 0,7 & 0,7 \\
\hline p05 & 2,2 & 1,5 & 1,6 & 1 & 2,1 & 1,7 \\
\hline p95 & 4 & 3,8 & 4 & 3,9 & 4,6 & 4,3 \\
\hline Asimetría & $-0,07$ & $-0,2$ & $-0,2$ & 0,04 & $-0,35$ & $-0,4$ \\
\hline
\end{tabular}

Fuente: elaboración propia

A continuación, se muestran las diferentes estrategias de trabajo autónomo según el año de cursada (véase tabla 2). Se evidencia que la estrategia de preparación de exámenes y las estrategias de participación suelen ir declinando con el avance del tiempo; las demás estrategias de trabajo autónomo se muestran oscilantes con el avance del tiempo. 
TABLA 2.

Estrategias de trabajo autónomo según año de cursada

\begin{tabular}{|c|c|c|c|c|c|c|c|}
\hline & Año & $\begin{array}{r}\text { Estrategias de } \\
\text { ampliación }\end{array}$ & $\begin{array}{l}\text { Estrategias de } \\
\text { colaboración }\end{array}$ & $\begin{array}{r}\text { Estrategias de } \\
\text { conceptualización }\end{array}$ & $\begin{array}{l}\text { Estrategias de } \\
\text { planificación }\end{array}$ & $\begin{array}{c}\text { Estrategias de } \\
\text { preparación } \\
\text { de exámenes }\end{array}$ & $\begin{array}{l}\text { Estrategias de } \\
\text { participación }\end{array}$ \\
\hline \multirow{3}{*}{ Primero } & Media & 3,1737 & 2,8316 & 3,1026 & 2,7895 & 3,7184 & 3,4684 \\
\hline & $\mathrm{N}$ & 38 & 38 & 38 & 38 & 38 & 38 \\
\hline & Desviación estándar & 0,58434 & 0,57802 & 0,62665 & 0,86111 & 0,68294 & 0,73415 \\
\hline \multirow{3}{*}{ Segundo } & Media & 3,0467 & 2,4333 & 2,8667 & 2,3200 & 3,6000 & 3,2200 \\
\hline & $\mathrm{N}$ & 15 & 15 & 15 & 15 & 15 & 15 \\
\hline & Desviación estándar & 0,66210 & 0,72964 & 0,77613 & 0,86123 & 0,57196 & 0,61667 \\
\hline \multirow{3}{*}{ Tercero } & Media & 3,4556 & 3,0000 & 3,2667 & 2,9333 & 3,5167 & 3,1000 \\
\hline & $\mathrm{N}$ & 18 & 18 & 18 & 18 & 18 & 18 \\
\hline & Desviación estándar & 0,71966 & 0,69197 & 0,83525 & 0,87850 & 0,75166 & 0,86501 \\
\hline \multirow{3}{*}{ Cuarto } & Media & 3,4083 & 2,9250 & 2,8667 & 2,6167 & 3,5250 & 2,9917 \\
\hline & $\mathrm{N}$ & 12 & 12 & 12 & 12 & 12 & 12 \\
\hline & Desviación estándar & 0,59001 & 0,80354 & 0,74142 & 0,59365 & 0,79673 & 0,51072 \\
\hline \multirow{3}{*}{ Quinto } & Media & 3,2250 & 2,9583 & 2,8167 & 2,3167 & 3,4333 & 2,9750 \\
\hline & $\mathrm{N}$ & 12 & 12 & 12 & 12 & 12 & 12 \\
\hline & Desviación estándar & 0,54793 & 0,80392 & 0,81668 & 0,82884 & 0,67600 & 0,78291 \\
\hline \multirow{3}{*}{ Total } & Media & 3,2432 & 2,8284 & 3,0305 & 2,6611 & 3,6011 & 3,2368 \\
\hline & $\mathrm{N}$ & 95 & 95 & 95 & 95 & 95 & 95 \\
\hline & Desviación estándar & 0,62410 & 0,69461 & 0,73428 & 0,84756 & 0,68812 & 0,74034 \\
\hline
\end{tabular}

En la tabla 2 se observa que los estudiantes de tercer año son quienes más usan las estrategias de ampliación, colaboración, conceptualización y planificación; mientras que los estudiantes de primer año son quienes más implementan las estrategias de preparación de exámenes y participación.

\section{Estrategia de ampliación}

Las estrategias de ampliación fueron el segundo grupo de estrategias más utilizadas por los estudiantes encuestados. En este grupo se incluyen afirmaciones, como: busco más información navegando por internet; realizo actividades complementarias; completo el estudio con lecturas/trabajos complementarios; elaboro una base de datos con toda la información obtenida en el desarrollo del trabajo; busco datos, relativos al tema, en internet; consulto bibliografía recomendada; preparo los exámenes teniendo en cuenta todo el material, no solo mis apuntes; consulto otros materiales bibliográficos o páginas de internet que ayuden o mejoren la comprensión o cuando me surgen dudas, o para ampliar algún concepto, realizo búsquedas en libros o en internet.

La pregunta con menor puntuación fue la relativa a "elaboro una base de datos con toda la información obtenida en el desarrollo del trabajo" y la mejor puntuada fue "cuando me surgen dudas, o para ampliar algún concepto, realizo búsquedas en libros o en internet". No se encontraron diferencias significativas respecto del sexo, la tenencia de hijos, el trabajo o el promedio de notas de los alumnos y el uso de las estrategias de ampliación $(\mathrm{p}>0,05)$.

\section{Estrategias de colaboración}

Se encontró un promedio de 2,8 en el uso de este grupo de estrategias (DE: 0,69). Se incluye en este grupo de estrategias afirmaciones como: conozco y utilizo los recursos que proporciona el campus; en la elaboración de resúmenes de cada uno de los temas integro las aportaciones hechas por otros compañeros en clase; intercambio los resúmenes de los temas con los compañeros; me organizo con los compañeros para pedir libros a la biblioteca; cuando descubro aportaciones nuevas en documentos complementarios a la bibliografía recomendada, lo comparto con los compañeros; intercambio con compañeros documentos, direcciones de webs que nos puedan ser útiles para el desarrollo de las actividades; consulto con los compañeros las dudas que se me plantean en el estudio del tema; reparto con algunos compañeros los libros de la bibliografía básica, elaborando sinopsis de cada uno de ellos, para compartirlos; pongo a disposición de los compañeros los apuntes que he elaborado para facilitar el estudio del temario; trabajo en colaboración para resolver un problema o investigar algo y reviso los apuntes de los compañeros para ver si aclaran las dudas. 
La pregunta menor puntuada fue la concerniente a "conozco y utilizo los recursos que proporciona el campus"; en tanto que la mejor puntuada fue "consulto con los compañeros las dudas que se me plantean en el estudio del tema". Finalmente, no se encontraron diferencias significativas respecto del sexo, la tenencia de hijos, el trabajo, el promedio de notas de los alumnos y el uso de las estrategias de colaboración $(\mathrm{p}>0,05)$.

\section{Estrategias de conceptualización}

El comportamiento general de este factor arrojó un promedio de 3,03 (DE: 0,73). Se incluye en este grupo de estrategias afirmaciones como: estudio con esquemas, resúmenes y cuadros sinópticos los contenidos de cada tema; cuando inicio la lectura de un tema, escribo notas que posteriormente me sirven de síntesis de lo leído; construyo una síntesis personal de los contenidos; realizo mapas conceptuales y esquemas globales; realizo un mapa conceptual con los conceptos más importantes de cada apartado; leo y esquematizo los contenidos; confecciono un resumen de cada tema y recopilo los contenidos que considero más importantes a modo de notas de estudio.

La pregunta menor puntuada fue la relacionada con "realizo un mapa conceptual con los conceptos más importantes de cada apartado"; entre tanto la pregunta mejor puntuada es la relacionada con "confecciono un resumen de cada tema". No se encontraron diferencias significativas respecto del sexo, la tenencia de hijos, el trabajo, el promedio de notas de los alumnos y el uso de las estrategias de conceptualización $(\mathrm{p}>0,05)$.

\section{Estrategias de planificación}

El comportamiento general de este factor arrojó un promedio de 2,66 (DE: 0,84). Este fue el factor con menor puntuación promedio. Se incluye en este grupo de estrategias afirmaciones como: al empezar el cuatrimestre, hago por escrito un plan de trabajo, reflejando el tiempo que dedicaré a cada asignatura y la fecha de los exámenes; planifico los tiempos y estrategias de estudio; evalúo el proceso de aprendizaje final; reparto el tiempo para el estudio de contenidos y la elaboración de las trabajos de cada tema, y planifico el tiempo de que dispongo para cada asignatura y trabajo práctico.

El ítem con más baja calificación fue "al empezar el semestre, hago por escrito un plan de trabajo, reflejando el tiempo que dedicaré a cada asignatura y la fecha de los exámenes”; mientras que el mejor puntuado fue "reparto el tiempo para el estudio de contenidos y la elaboración de trabajos de cada tema”. No se encontraron diferencias significativas respecto del sexo, la tenencia de hijos, el trabajo, el promedio de notas de los alumnos y el uso de las estrategias de planificación $(\mathrm{p}>0,05)$.

\section{Estrategias de preparación de exámenes}

Este fue el grupo de estrategias mejor evaluadas del constructo con un promedio de 3,6 (DE: 0,68). Se incluye en este grupo de estrategias afirmaciones, como: leo todo el material de la asignatura y hago una selección de los puntos más importantes para trabajarlos; cuando hay debate, tengo en cuenta las aportaciones de los compañeros para realizar la mía; antes de los exámenes dedico unos días de repaso para aclarar dudas finales; para preparar el examen me baso principalmente en los aspectos que el profesor marca como importantes; repaso las indicaciones que el profesor nos ha dado a lo largo del curso, y realizo una primera lectura rápida y después otra más detenida con copia o trascripción de lo más relevante.

La pregunta con menor puntaje promedio fue "cuando hay debate, tengo en cuenta las aportaciones de los compañeros para realizar la mía"; en tanto que la mejor puntuada fue "para preparar el examen me baso principalmente en los aspectos que el profesor marca como importantes". No se encontraron diferencias 
significativas respecto del sexo, la tenencia de hijos, el trabajo, el promedio de notas de los alumnos y el uso de las estrategias de preparación de exámenes ( $\mathrm{p}>0,05)$.

\section{Estrategias de participación}

La pregunta con menor puntuación fue "anoto mis dudas para consultarlas más a fondo en una segunda lectura”; mientras que la mejor puntuada fue "sigo, aprovecho y participo en clases". Se incluye en este grupo de estrategias afirmaciones como: tomo nota de las respuestas del profesor a las dudas propias o de los compañeros; anoto mis dudas para consultarlas más a fondo en una segunda lectura; aclaro las dudas con el profesor en clase o en tutoría; respondo a las preguntas planteadas en clase; corrijo las actividades propuestas para comprobar mis conocimientos, y sigo, aprovecho y participo en las clases.

No se encontraron diferencias significativas respecto del sexo, la tenencia de hijos, el trabajo y el uso de las estrategias de preparación de exámenes $(p>0,05)$. Por otro lado, se identificaron diferencias estadísticamente significativas relativas al promedio de notas del alumno y el uso de las estrategias de participación ( $\mathrm{p}=$ 0,0003); hallándose en los estudiantes con mayor promedio de nota (de 8 a $9 / 10)$ un mayor de uso de este grupo de estrategias.

En síntesis, no se encontraron diferencias significativas respecto del sexo, la tenencia de hijos, el trabajo o el promedio de notas de los alumnos y el uso de las estrategias de ampliación ( $p>0,05)$. En ninguna de las estrategias de aprendizaje autónomo investigadas, excepto en las estrategias de participación, donde los estudiantes con mejores notas presentaban un mayor uso de estas.

\section{Discusión}

Las estrategias que facilitan el aprendizaje autónomo son un método eficaz para adquirir y desarrollar habilidades, a efectos de planificar las tareas, preparar los exámenes, organizar el tiempo, aumentar la motivación para aprender, maximizar el aprendizaje y mejorar el rendimiento académico del estudiantado.

A fin de alcanzar las competencias necesarias para desempeñar su futura labor profesional, los estudiantes de la carrera de Kinesiología deben poner en práctica una amplia variedad de estrategias de aprendizaje; al mismo tiempo, el desarrollo de las estrategias hace parte y es el resultado de la adquisición de competencias mismas.

Es necesario destacar que el proceso de aprendizaje requiere que el alumno tome una posición activa y responsable en su proceso de formación. Lo anterior y dado el nivel de exigencias del ámbito universitario, se condice con lo hallado en el grado de implementación de esta estrategia de ampliación (8).

Resulta particularmente interesante el hecho de que las estrategias de colaboración tengan poca implementación. Se encuentra una comunidad académica poco organizada para el trabajo en grupo. Esta estrategia de aprendizaje no se opone al trabajo individual, sino que es una herramienta complementaria que fortalece y favorece el desarrollo global del alumno (9). Estos métodos comparten la idea de que los estudiantes trabajan juntos para aprender y les da un rol importante en el proceso de aprendizaje más allá del propio, sino también sobre el de sus compañeros.

De los datos encontrados puede inferirse que los estudiantes les dan mucha importancia a los modelos evaluativos implementados por los docentes, siendo las estrategias de preparación de exámenes las mayor puntuadas. Datos similares se describen en grupos en la bibliografía consultada (10).

En cuanto a las estrategias de participación, vale destacar la relación de la participación en clases con la calificación final del alumno. Hacer parte al alumno de su proceso instruccional también debe motivarse desde el rol docente, impulsándolo a aprender y participar de las actividades propuestas y diseñadas, porque ello 
maximiza sus saberes. Autores como Vigotzky resaltaban la importancia de la socialización en los procesos de aprendizaje, pues este es de gran importancia en la adquisición de conocimientos (11).

Es necesario reflexionar y repensar la labor docente, apoyando el proceso de aprendizaje hacia la autonomía, pues es un desafío que prevalece sobre las labores de instrucción tradicionales, que centran el proceso de enseñanza en el docente y dejan relegado al discente (12).

Identificar estas estrategias implementadas para el aprendizaje puede ser de gran ayuda en la búsqueda de mejorar el rendimiento académico, y se hace necesario dentro de la gestión educativa considerar diagnosticar y conocer el estilo predilecto para adquirir y procesar la información por parte del estudiantado, con el fin de personalizar la enseñanza y diseñar estrategias efectivas de aprendizaje, teniendo en cuenta que la manera en que estos aprenden es propia y no impuesta (13).

Se hace hincapié en la necesidad de fomentar el desarrollo del compromiso del estudiante con su aprendizaje y su actuación profesional, buscando que aprenda de manera autónoma, independiente y permanente, aun sin la presencia de un docente (14).

Finalmente, aun cuando la investigación permitió conocer la realidad específica de la población investigada, se propone que se planteen más estudios relacionados con población en similitud de condiciones y que se indague por las estrategias comúnmente implementadas y cómo impacta su uso en el desempeño académico del estudiante, favoreciendo el desarrollo de estas últimas desde el rol docente.

Como limitación, el presente trabajo cuenta con la participación de una muestra intencional, por lo cual las características propias del muestreo inducen sesgos relacionados con la muestra, como el sesgo de inclusión, en el cual sus características pueden o no ser representativas de la población general.

\section{Conclusiones}

\footnotetext{
- En términos generales, se puede concluir que:

- Los estudiantes de la carrera de Kinesiología y Fisiatría presentan un bajo uso de las estrategias de planificación y un amplio desarrollo de las estrategias de preparación de exámenes.

- Solo las estrategias de participación mostraron estar relacionadas con el rendimiento académico en la muestra del presente estudio, siendo implementadas en mayor medida por los estudiantes con mejor rendimiento académico.
}

\section{Referencias}

1. Lobato C. Estudio y trabajo autónomo del estudiante. En Miguel M, coordinador. Metodologías de enseñanza y aprendizaje para el desarrollo de competencias: Orientaciones para el profesorado universitario ante el Espacio Europeo de Educación Superior. Madrid: Alianza; 2006. pp. 191-223.

2. López-Aguado M. Estrategias de aprendizaje en estudiantes universitarios: Diferencias por género, curso y tipo de titulación. TESI. 2011;12(2):203-33.

3. Fernández E, Fernández M. El Espacio Europeo de Educación Superior. En Benítez JL, Berbén ABJ, Justicia F y de la Fuente J, coordinadores. La universidad ante el reto del espacio europeo de educación superior: investigaciones recientes. Madrid: EOS; 2006. pp. 17-47.

4. Dewey J. Democracy and education: An introduction to the philosophy of education. New York: Free Press; 1916.

5. Álvarez B, González Mieres C, García Rodríguez N. La motivación y los métodos de evaluación como variables fundamentales para estimular el aprendizaje autónomo. Revista de Docencia Universitaria. 2007;1(2):1-12.

6. Márquez C, Fasce E, Pérez C, Ortega J, Parra P, Ortiz L, et al. Aprendizaje autodirigido y su relación con estilos y estrategias de aprendizaje en estudiantes de medicina. Rev Med Chile. 2001;142(11):1422-30.

7. López-Aguado M. Diseño y análisis del Cuestionario de Estrategias de Trabajo Autónomo (CETA) para estudiantes universitarios. Rev Psic. 2010;15(1):77-99. 
8. Hernández Díaz A, González Hernández M. Estrategias de aprendizaje en la formación universitaria. EDUCERE. 2015;19(63):441-54.

9. Johnson DW, Johnson R, Holubec E. Circles of learning. 4th ed. Edina, MN: Interaction Book Company; 1993.

10. Uribe Meneses A. Características del aprendizaje autónomo de los estudiantes del programa de enfermería de la Universidad de Pamplona. Rev Cien y Cuid. 2012;9(1):24-33.

11. Vigotzky L. Obras escogidas. Tomos I y III. Ed. Madrid: Visor; 1995.

12. Escribano González A. Aprendizaje cooperativo y autónomo en la enseñanza universitaria. Enseñanza. 1995;13:89-102.

13. Espinoza-Freire E, Serrano Polo O, Brito Paredes P. El trabajo autónomo en estudiantes de la universidad de la universidad técnica de Machala. Univ Soc. 2017;9(2):202-12.

14. Vives-Varela T, Durán-Cárdenas C, Varela-Ruíz M, Fortoul van der Goes T. La autorregulación en el aprendizaje, la luz de un faro en el mar. Inv Ed Med. 2014;3(9):34-9.

\section{Notas}

* Artículo de investigación

1. Conflictos de interés: los autores declaran no tener conflictos de interés.

2. Financiación: el presente estudio fue financiado por los autores.

\section{Licencia Creative Commons CC BY 4.0}

Cómo citar este artículo: Canova Barrios C, Pecker L. Características del aprendizaje autónomo en estudiantes de kinesiología de una institución de educación superior de Buenos Aires. Investig Enferm Imagen Desarr. 2020;21(2). https://doi.org/10.11144/Javeriana.ie21-2.caae 